\title{
Selective inhibition of PI3K110 $\alpha$ as a novel therapeutic strategy for cetuximab-resistant oral squamous cell carcinoma
}

\author{
HIROKI TSUCHIHASHI $^{1}$, TOMOFUMI NARUSE ${ }^{1}$, SOUICHI YANAMOTO $^{1}$, KOHEI OKUYAMA ${ }^{1,2}$, \\ KOHEI FURUKAWA $^{1}$, KEISUKE OMORI ${ }^{1}$ and MASAHIRO UMEDA ${ }^{1}$ \\ ${ }^{1}$ Department of Clinical Oral Oncology, Graduate School of Biomedical Sciences, \\ Nagasaki University, Nagasaki 852-8588; ${ }^{2}$ Department of Oral and Maxillofacial Surgery, \\ Graduate School of Medical and Dental Sciences, Tokyo Medical and Dental University, Tokyo 113-8549, Japan
}

Received August 21, 2019; Accepted March 31, 2020

DOI: $10.3892 /$ or.2020.7674

\begin{abstract}
High expression of the $110 \mathrm{kDa}$ catalytic subunit of the class IA PI3K (PI3Kp110 $\alpha$ ) may play an important role in cetuximab resistance exhibited by both colorectal cancer and head and neck squamous cell carcinoma. Therefore, the present study aimed to examine the association between the expression of proteins in the PI3Kp110 $\alpha$ pathway and cetuximab resistance, and the antitumor effects of alpelisib (PI3K inhibitor) and cetuximab in oral squamous cell carcinoma (OSCC) cells. The association between PI3Kp110 $\alpha$ protein expression levels and the tumor response to cetuximab was determined using immunohistochemistry. OSCC cells were treated with alpelisib, cetuximab, or in combination, and the effects were examined in vitro and in vivo. $\mathrm{PI} 3 \mathrm{Kp} 110 \alpha$ protein expression was significantly associated with the tumor response to cetuximab $(\mathrm{P}<0.05)$ and 1 -year progression-free survival and overall survival $(\mathrm{P}<0.05)$. Combined treatment of alpelisib and cetuximab resulted in enhanced antitumor effects in vitro compared with either agent administered alone. In particular, the expression level of $\mathrm{N}$-cadherin, an epithelial-mesenchymal transition-related protein, was decreased, suggesting that the invasion potential of cetuximab-resistant cells decreased. Furthermore, the expression of proteins in the PI3K pathway were decreased in tumors from mice with OSCC xenografts treated with alpelisib and cetuximab in combination. These results indicate that novel regimens of systemic therapy (such as chemotherapy), with combinations of cetuximab and alpelisib, may be beneficial for patients with cetuximab-resistant OSCC.
\end{abstract}

Correspondence to: Dr Tomofumi Naruse, Department of Clinical Oral Oncology, Graduate School of Biomedical Sciences, Nagasaki University, 1-7-1 Sakamoto, Nagasaki 852-8588, Japan

E-mail: natsuaya1023@gmail.com

Key words: PIK3CA gene, PI3Kp110 , alpelisib, cetuximab, PI3K pathway

\section{Introduction}

Over the last 30 years, systemic therapeutic (drug therapy) guidelines for patients with recurrent or metastatic $(\mathrm{R} / \mathrm{M})$ head and neck squamous cell carcinoma (HNSCC) have not changed (1). In 2008, the Extreme trial (2) found that the addition of cetuximab (trade name Erbitux ${ }^{\mathrm{TM}}$ ), a molecular targeted agent against epidermal growth factor receptor (EGFR), to the standard platinum and 5-fluorouracil (5-FU) regimen improved median overall survival (OS) and progression-free survival (PFS) in HNSCC. The National Comprehensive Cancer Network (NCCN) Clinical Practice Guidelines have also suggested that systemic therapy, including cetuximab, should be listed as the recommended category $2 \mathrm{~B}$ for its effectiveness in treating very advanced HNSCC (3). Based on this evidence, cetuximab therapy was selected for $\mathrm{R} / \mathrm{M}$ oral squamous cell carcinoma (OSCC), and the tumor growth was controlled for unresectable R/M OSCC (4,5). However, some OSCC tumors acquired resistance with long-term cetuximab administration, and new lesions appeared in the brain following 2 years of treatment (6); thus management of these lesions is a future priority.

Although cetuximab is reported to have significant therapeutic efficacy against $\operatorname{HNSCC}(2,7,8)$, the phosphatidylinositol-4,5-bisphosphate 3-kinase catalytic subunit $\alpha$ isoform (PIK3CA) gene is a candidate gene involved in the acquired resistance to long-term cetuximab treatment due to point mutations within the gene (9-11). PIK3CA, a key element of the PI3K/AKT pathway (Fig. 1), is located on chromosomal $3 q 26.3$, encoding the $110 \mathrm{kDa}$ catalytic subunit of class IA PI3K (PI3Kp110 $\alpha)(12,13)$. Somatic PIK3CA gene point mutations have been identified as cancer-specific or heterozygous, and these mutations have been proven to activate the PI3K/Akt-mTOR signaling pathway in human cancers, including HNSCC $(12,13)$. Most somatic PIK3CA gene mutations are clustered in the helical domain encoded by exon 9 and the kinase domain encoded by exon 20 in OSCC $(12,13)$. In addition, three hotspot mutations in these exons, E542K, E545K and H1047R, were proven to activate the PI3K/AKT pathway through the phosphorylation of AKT $(12,13)$. Overexpression of the mutant PIK3CA gene markedly increased Akt/mTOR pathway activation compared with that in overexpressed 
wild-type PIK3CA in HNSCC (12). Consequently, the PIK3CA point mutation may promote cetuximab resistance in HNSCC $(8,9)$. Copy number amplifications and gene point mutations of PIK3CA have also been reported in OSCC $(12,14)$ and observed in advanced stages of OSCC (12). To the best of our knowledge, regarding clinical outcomes of PIK3CA mutations and cetuximab therapy, PIK3CA point mutations in colorectal cancer are associated with clinical resistance to EGFR-targeted monoclonal antibodies (cetuximab or panitumumab) $(15,16)$ however, the clinical outcome of PIK3CA mutants against OSCC has not been investigated.

Therefore, the present study aimed to determine the clinicopathological significance of the tumor response to cetuximab therapy in R/M OSCC. Furthermore, the antitumor effects of PI3Kp110 inhibitor, alone or in combination with cetuximab, were examined to clarify the additional benefit of combination therapy in the preclinical model of OSCC.

\section{Materials and methods}

Patients. The present study was approved by the independent Ethics Committee of Nagasaki University Hospital (approval no. 19081915). The medical records of 25 patients who received cetuximab therapy for R/M OSCC between December 2012 and March 2017 were retrospectively reviewed at Nagasaki University Hospital (Table I). The Eastern Cooperative Oncology Group (ECOG) (17) performance status score was used as follows: 0, Fully active, able to perform all pre-disease performance without restriction; 1 , restricted in physically strenuous activity but ambulatory and able to perform light or sedentary work, e.g., light house work and office work, 2 , ambulatory and capable of all selfcare but unable to perform any work activities for example walking about $>50 \%$ of waking hours. Survival data was reviewed in March 2019. Furthermore, 4- $\mu$ m paraffin-embedded sections (fixed in buffered $10 \%$ formalin for $24 \mathrm{~h}$ at room temperature) from biopsy or resected specimens obtained immediately prior to cetuximab therapy were obtained. Tumor response was assessed every 4-8 weeks with repeated clinical and enhanced computed tomography assessments. in accordance with the RECIST guidelines (18). Normal oral mucosal specimens were obtained from 10 healthy individuals undergoing routine surgical removal of their third molars during the aforementioned study period at Nagasaki University Hospital. The tumor stage was classified according to the TNM classification of the Union for International Cancer Control (19). Tumor histological differentiation was defined according to the World Health Organization classification (20). The pattern of invasion was assessed according to Bryne's classification (score 1, well-defined margins; score 2, solid strings and/or inlets; score 3 , small cell groups $n<15$; score 4 , cellular diffuse dissociation and characterized in small and/or isolated cell groups) (21).

Immunohistochemistry staining and evaluation. Sections were deparaffinized in xylene, rehydrated in a descending alcohol series (70-100\%), incubated in $10 \mathrm{mM}$ citrate buffer (pH 6.0), and heated at $121^{\circ} \mathrm{C}$ for $5 \mathrm{~min}$ for antigen retrieval. Endogenous peroxidases were blocked by incubation with $0.3 \% \mathrm{H}_{2} \mathrm{O}_{2}$ in methanol for $30 \mathrm{~min}$ at room temperature.
Immunohistochemistry staining was performed using the EnVision kit (EnVision+; Dako; Agilent Technologies, Inc.). The PI3Kp110 $\alpha$ (cat. no. 4249S; dilution 1:400; Cell Signaling Technology, Inc.) rabbit polyclonal primary antibody was used. Tissue sections were washed in phosphate-buffered saline, followed by incubation with the primary antibody overnight at $4^{\circ} \mathrm{C}$, then with a secondary antibody (cat. no. K4003; pre-diluted; Dako; Agilent Technologies, Inc.) for $30 \mathrm{~min}$. Reaction products were visualized by immersing the sections in diaminobenzidine solution. The samples were counterstained with Meyer's hematoxylin for $10 \mathrm{~min}$ at room temperature and subsequently mounted.

The expression of PI3Kp110 $\alpha$ protein was evaluated by calculating the total immunostaining score as the product of the number of positive cells (proportional score) and intensity scores at the invasion front (inside surface) of the tumor in two fields of view. The proportional scores were based on the calculated fraction of positively stained tumor cells $(0$, none; $1,<10 \% ; 2,10-50 \% ; 3,50-80 \% ; 4,>80 \%)$. The intensity score represented the calculated staining intensity $(0$, no staining; 1 , weak; 2 , moderate; 3 , strong). Total immunostaining scores ranged from 0 to 12 , with positive cases defined as total scores $>4$. Patient samples showed a bimodal distribution of immunohistochemistry protein expression, with the most common score of 3-4, therefore the cut-off for positive expression was set to $>4$. All immunohistochemical assessments were performed by both an oral cancer surgeon and an oral cancer pathologist, who were blinded to the samples.

Cell lines and reagents. A total of three human OSCC cell lines, SAS (wild-type PIK3CA gene), HSC-2 and HSC-3 (both with mutant PIK3CA gene) were used in the present study, as found in a previous study (12) and were cultured in a 1:1 mixture of Ham's F-12/Dulbecco's modified Eagle's medium supplemented with $10 \%$ fetal bovine serum (all Trace Scientific, Ltd; Thermo Fisher Scientific, Inc.). All cells were maintained at $37^{\circ} \mathrm{C}$ in a humidified incubator with $5 \% \mathrm{CO}_{2}$ and $19 \% \mathrm{O}_{2}$. Alpelisib, a selective PI3K $\alpha$ inhibitor, was purchased from Selleck Chemicals (cat. no. BS119QD), dissolved in dimethyl sulfoxide (DMSO), and adjusted to a range of concentrations (0.1-100 $\mu \mathrm{M})$ with culture medium (1:1 mixture of Ham's F-12 and DMEM supplemented with $10 \%$ fetal bovine serum) (all Trace Scientific, Ltd; Thermo Fisher Scientific, Inc.). Cetuximab (cat. no. C225) was purchased from Merck KGaA. Working solutions were freshly prepared from the stock solution by diluting with cell culture medium on the day of the experiment.

Cell cytotoxicity assay. Cells were seeded in 96-well plates at a concentration of $1.5 \times 10^{3}$ per well and incubated for $24 \mathrm{~h}$. Cells were exposed to alpelisib or cetuximab at concentrations ranging from 0.1 to $100 \mu \mathrm{M}$. At the end of the 72-h treatment, cells were incubated with $0.5 \mathrm{mg} / \mathrm{ml}$ MTT (Sigma-Aldrich; Merck KGaA). The medium was replaced with $100 \mu 1$ DMSO and vortexed for $10 \mathrm{~min}, 4 \mathrm{~h}$ later. Absorbance was recorded at $570 \mathrm{~nm}$, using a microplate auto reader (Multiskan FC; Thermo Fisher Scientific, Inc.). Cell viability was calculated as the percentage of cells from that in the control group, which received medium only. IC50 is a widely used indicator of the effectiveness of inhibitors in pharmaceutical research (22) 


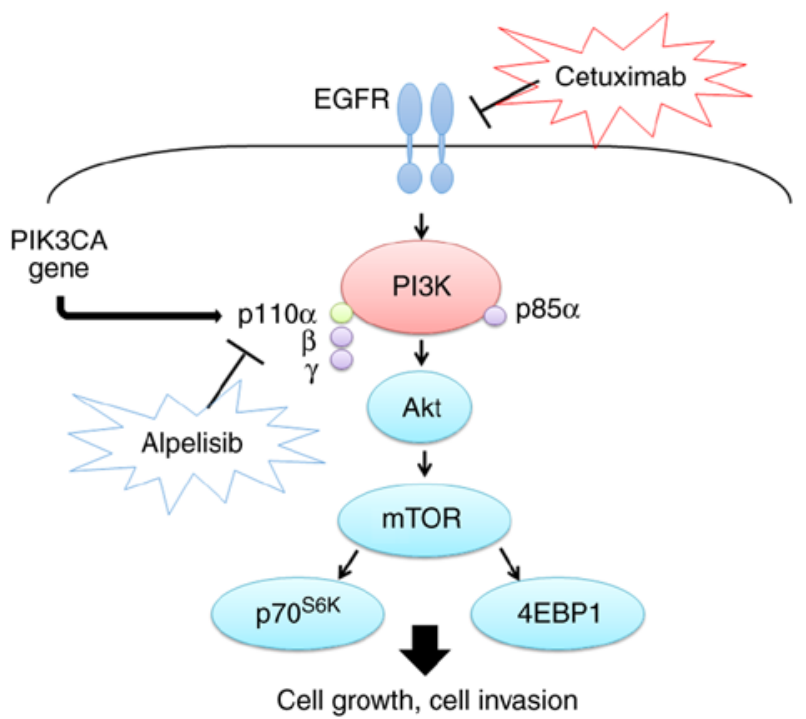

Figure 1. Mechanisms of the PI3K pathway in cancer cells. Cetuximab is a molecular targeted agent against epidermal growth factor receptor, while alpelisib is a selective PI3K $\alpha$ inhibitor.

and was subsequently calculated from the results. $\mathrm{IC}_{50}$ was calculated from the results of MTT assay, using the following equation, $\mathrm{IC}_{50}=10^{\wedge}[\log (\mathrm{A} / \mathrm{B}) \times(50-\mathrm{C}) /(\mathrm{D}-\mathrm{C})+\log (\mathrm{B})]$ was used. A indicates high concentration across $50 \%$; $\mathrm{B}$, indicates low concentration across $50 \%$; $\mathrm{C}$, indicates inhibition rate at $\mathrm{B}$ and $\mathrm{D}$ indicates inhibition rate at $\mathrm{A}$. Therefore, the appropriate concentration was used to compare the efficacy of alpelisib and cetuximab with that in the control group.

Western blot analysis. Cells were harvested using trypsinization, washed with PBS, and precipitated by centrifugation $\left(4^{\circ} \mathrm{C} ; 22,140 \mathrm{x} \mathrm{g}\right.$, for $\left.5 \mathrm{~min}\right)$. The Mammalian Cell Extraction kit (BioVision, Inc.) was used to extract the total proteins. All subsequent manipulations were performed on ice. Cells were incubated in Extraction Buffer Mix. The protein concentration of each sample was measured using the micro-bicinchoninic acid protein assay (Pierce; Thermo Fisher Scientific, Inc.). After the samples were denatured in SDS sample buffer, they were incubated at $70^{\circ} \mathrm{C}$ for $10 \mathrm{~min}$ and then $10 \mu \mathrm{g}$ was loaded onto a 4-12\% NuPAGE NOVEX bis-tris polyacrylamide gel or a $3-8 \%$ NuPAGE NOVEX tris-acetate polyacrylamide gel. After electrophoresis, the separated proteins were transferred to iBlot polyvinylidene difluoride membranes, using the iBlot Dry Blotting System (Thermo Fisher Scientific, Inc.). The membrane was blocked for 30 min using blocking reagent (part A and B) from the Western Breeze Immunodetection kit (Thermo Fisher Scientific, Inc.) at room temperature. Primary antibodies against PI3Kp110 $\alpha$ (cat. no. 4249S; Cell Signaling Technology, Inc.), Akt (cat. no. 9272S; Cell Signaling Technology, Inc.), phosphorylated (p)-Akt (cat. no. 4060S; Cell Signaling Technology, Inc.), mTOR (cat. no. 2972S; Cell Signaling Technology, Inc.), p-mTOR ${ }^{\text {Ser2448 }}$ (cat. no. 2971S; Cell Signaling Technology, Inc.), p70S6K (cat. no. 9202S; Cell Signaling Technology, Inc.), p-p70S6K ${ }^{\text {Tyr389 }}$ (cat. no. 9205S; Cell Signaling Technology, Inc.), 4EBP-1 (cat. no. 9452S; Cell Signaling Technology, Inc.), p-4EBP1 (cat. no. 9459S; Cell Signaling Technology, Inc.), E-cadherin, and N-cadherin
Table I. Clinicopathological characteristics of the 25 patients with oral squamous cell carcinoma.

\begin{tabular}{|c|c|}
\hline Characteristics & Value \\
\hline \multicolumn{2}{|l|}{$\operatorname{Sex}, \mathrm{n}(\%)$} \\
\hline Male & $11(44.0)$ \\
\hline Female & $14(56.0)$ \\
\hline Median age, years & 68 \\
\hline Age, range, years & $50-90$ \\
\hline \multicolumn{2}{|l|}{ Primary site, n (\%) } \\
\hline Tongue & $8(32.0)$ \\
\hline Gingiva & $14(56.0)$ \\
\hline Oral floor & $1(4.0)$ \\
\hline Intraosseous & $1(4.0)$ \\
\hline Buccal & $1(4.0)$ \\
\hline \multicolumn{2}{|l|}{ Differentiation, n (\%) } \\
\hline Well & $22(88.0)$ \\
\hline Moderate & $1(4.0)$ \\
\hline Poor & $1(4.0)$ \\
\hline Not specified & $1(4.0)$ \\
\hline \multicolumn{2}{|l|}{ ECOG Performance status, n (\%) } \\
\hline 0 & $13(52.0)$ \\
\hline 1 & $9(36.0)$ \\
\hline 2 & $3(12.0)$ \\
\hline \multicolumn{2}{|l|}{ Initial treatment, $\mathrm{n}(\%)$} \\
\hline Surgery alone & $15(60.0)$ \\
\hline Surgery + adjuvant RT & $3(12.0)$ \\
\hline Surgery + adjuvant CCRT & $7(28.0)$ \\
\hline \multicolumn{2}{|l|}{ Cetuximab regimen, $\mathrm{n}(\%)$} \\
\hline Cet + RT & $15(60.0)$ \\
\hline Cet + PTX & $8(32.0)$ \\
\hline Cet + FP & $1(4.0)$ \\
\hline Cet alone & $1(4.0)$ \\
\hline Median number of treatment cycles, $n$ & 10 \\
\hline Number of treatment cycles, range, $n$ & $2-70$ \\
\hline
\end{tabular}

RT, radiotherapy; CCRT, concurrent chemoradiotherapy; PTX, paclitaxel; FP, 5-fluorouracil + cisplatin; ECOG, Eastern Cooperative Oncology Group.

(DAKO; Agilent Technologies, Inc.) were used at 1:1,000 dilution and the membrane was incubated for one $h$ at room temperature. $\beta$-actin (4970S; Cell Signaling Technology, Inc.) was used as the loading control. The secondary antibody (Western Breeze Immunodetection kit; Thermo Fisher Scientific, Inc.) was used at a dilution of 1:10 at room temperature for $30 \mathrm{~min}$. Signals were detected using the Western Breeze Immunodetection kit (Thermo Fisher Scientific, Inc.). PI3Kp110 $\alpha / \beta$-actin ratio was determined using Image $\mathbf{J}$ software v1.51 (National Institutes of Health).

Cell migration and invasion assay. The method used for migration and invasion was the same, in that a Biocoat Matrigel invasion chamber containing an internal chamber with an $8-\mu \mathrm{m}$ 

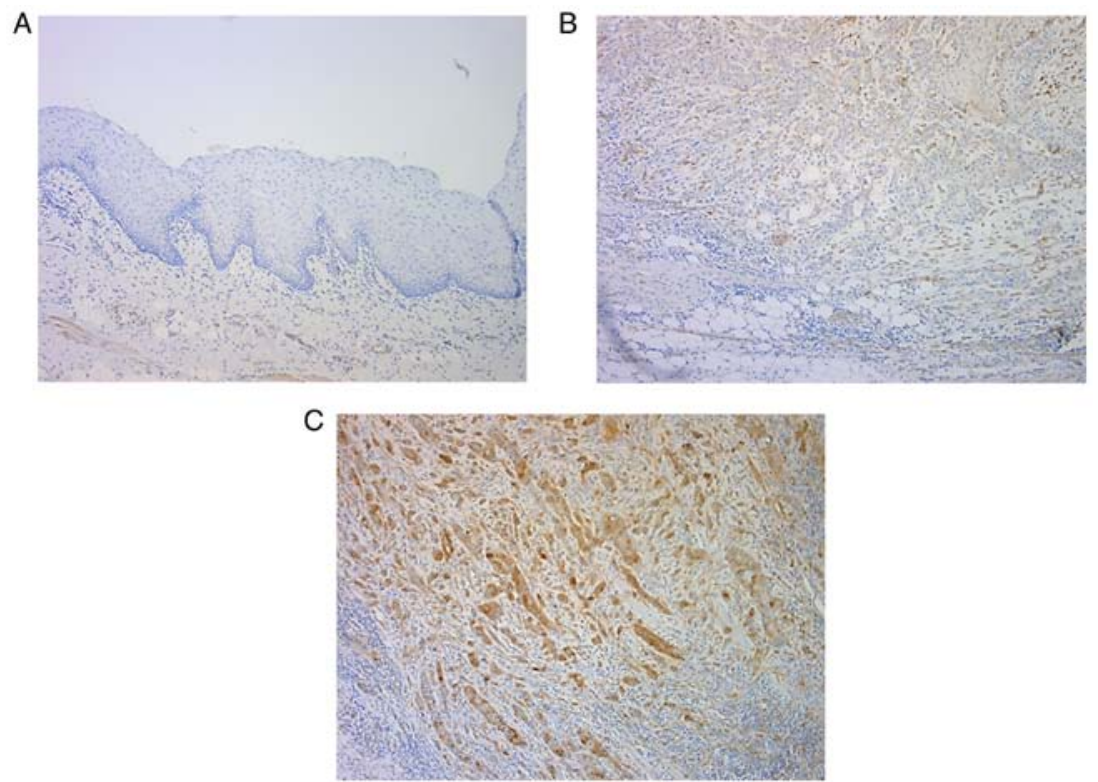

Figure 2. Representative immunohistochemical staining of PI3Kp110 $\alpha$. (A) Negative PI3Kp110 $\alpha$ staining is observed in normal epithelium. (B) Weak staining for PI3Kp110 $\alpha$ in OSCC with a Bryne's score of 3. (C) OSCC with Bryne's score of 3, demonstrates strong PI3Kp110 $\alpha$ cytoplasmic and nuclear expression (staining index of 12). Magnification x100.

porous membrane bottom was used for both assays; however, for the invasion assay the chamber was coated with Matrigel for $2 \mathrm{~h}$ at $37^{\circ} \mathrm{C}$ (Becton, Dickinson and Company). A total of 12 cell culture inserts and a 24-well multiwall companion plate were used for the experiment. The membranes were rehydrated with warm serum-free 1:1 mixture of Ham's F-12 and DMEM for $2 \mathrm{~h}$. Cells were collected using trypsinization, followed by seeding, at a density of $1.25 \times 10^{5}$, in the internal chamber with serum-free DMEM. The lower chamber was filled with medium containing $10 \%$ FBS as a chemoattractant. Cells were incubated for $72 \mathrm{~h}$, and non-invading, and non-migrating cells were removed from the top of the wells with a cotton swab, and cells that transferred to the inverse surface of the membrane were subjected to Diff-Quick staining for $15 \mathrm{sec}$ at room temperature. Cells were counted using a light microscope at x100 magnification. Cells that passed through a control chamber without Matrigel were used for calculating the migration index. The number of cells that passed through the Matrigel chamber were divided by the control cell count to calculate the percentage of invasion. All experiments were performed in triplicate, and cell numbers were counted in at least two fields of view/well.

Xenograft model assay. The animal protocol was approved by the Animal Care and Use Committee at Nagasaki University Graduate School of Biomedical Sciences (approval no. 1901041500). Briefly, a total of 16, female BALB/c-nu/nu mice (6 weeks old) weighing 18-20 g were purchased from Japan SLC Inc. and maintained in a barrier unit, under standard conditions (temperature, $20-26^{\circ} \mathrm{C}$; humidity, $40-70^{\circ} \mathrm{C}$; 12-h light/dark cycle; with ad libitum access to food and water). HSC-3 cells $\left(1 \times 10^{7}\right)$ were suspended in $100 \mu \mathrm{l}$ PBS and injected subcutaneously into the mice using a 21-gauge needle. After growing to $10-15 \mathrm{~mm}$ in diameter, the mice were sacrificed using carbon dioxide. When the flow rate had displaced $>30 \%$ of the volume of air in the chamber (infusion of carbon dioxide was $1 \mathrm{~min}$ ), the mice were checked for $>5 \mathrm{~min}$ and death was confirmed by observing lack of respiration and cardiac output. The HSC-3 tumor was extracted, cut into $1-\mathrm{mm}^{3}$ sections, and transplanted subcutaneously into the back of 12 different BALB/c-nu/nu mice. These mice were divided into four treatment groups: Control, cetuximab, alpelisib, and cetuximab plus alpelisib. The tumors were allowed to grow to 5-10 $\mathrm{mm}$ in diameter, following which the tumor-bearing mice were treated for 4 weeks with cetuximab $(20 \mathrm{mg} / \mathrm{kg}$, three times/week) and/or alpelisib (20 mg/kg, three times/week). Cetuximab was diluted 1:4 in saline, while alpelisib was dissolved in $64.5 \%$ saline, and the concentration was adjusted using $30 \%$ polyethylene glycol $400,0.5 \%$ polyoxyethylene sorbitan monooleate 80 , and $5 \%$ propylene glycol. The control group received saline only. All treatments were delivered by intraperitoneal injection. The mice were treated for 4 weeks, after which time the xenografted tumors were excised, fixed in buffered $10 \%$ formalin for $24 \mathrm{~h}$ at room temperature, and embedded in paraffin for histological examination using hematoxylin and eosin staining, and immunohistochemical examination using PI3Kp110 $\alpha$ (cat. no. 4249S; dilution 1:400), EGFR (cat. no. 4267S; dilution 1:100), and p-mTOR ${ }^{\text {Ser2448 }}$ (cat. no. 2976S; dilution 1:100) antibodies (all Cell Signaling Technology, Inc.), and incubated overnight at $4^{\circ} \mathrm{C}$. Sections (4- $\mu \mathrm{m}$ thick) were deparaffinized in xylene, rehydrated in a descending alcohol series (70-100\%), then incubated with Meyer's hematoxylin stain for $4 \mathrm{~min}$ and Eosin stain for $1 \mathrm{~min}$ at room temperature.

Statistical analysis. All statistical analyses were performed using Excel software v3.0 (Microsoft Corporation). Associations between the expression level of proteins of interest and clinicopathological characteristics were analyzed using Fisher's exact test. Continuous data are presented as mean \pm standard deviation. Survival analyses were calculated using the Kaplan-Meier method and compared using the log-rank test. The correlations 
Table II. Tumor response of cetuximab therapy and association between expression of $\mathrm{PI} 3 \mathrm{Kp} 110 \alpha$ and tumor response. $(n=25)$.

\begin{tabular}{lcc}
\hline Factor & Value & P-value \\
\hline Best response, n (\%) & & \\
CR & $9(36.0)$ & \\
PR & $8(32.0)$ & \\
SD & $4(16.0)$ & \\
PD & $4(16.0)$ & \\
Overall response ratea, \% & 68.0 & \\
Disease control rateb, \% & 84.0 \\
PI3Kp110 $\alpha$ & & \\
CR/PR, n & & \\
- & 10 & \\
+ & 7 & \\
SD/PD, n & & \\
- & 1 & \\
+ & 7 & \\
\hline
\end{tabular}

${ }^{\mathrm{a}} \mathrm{CR}+\mathrm{PR} .{ }^{\mathrm{b}} \mathrm{CR}+\mathrm{PR}+\mathrm{SD} . \mathrm{CR}$, complete response; PR, partial response; $\mathrm{SD}$, stable disease; $\mathrm{PD}$, progressive disease; - negative; + , positive.

between protein expression levels of PI3Kp110 $\alpha$ and cell migration and invasion, and with cetuximab sensitivity, in the OSCC cell lines, were analyzed using the Spearman's rank correlation test. A multiple comparison test between two groups in MTT assay, migration and invasion assays was performed using the Scheffe's method. $\mathrm{P}<0.05$ were considered to indicate a statistically significant result.

\section{Results}

Expression of $P I 3 K p 110 \alpha$ in OSCC. In normal oral epithelium, the expression of PI3Kp110 $\alpha$ was negative (Fig. 2A). Among the 25 patients with OSCC, PI3Kp110 $\alpha$ expression was detected in $56 \%$ of the patients using immunohistochemical staining. PI3Kp110 $\alpha$ was expressed primarily in the cytoplasm and nucleus of the tumor cells, and the staining intensity ranged from weak to strong (Fig. 2B and C).

Association of PI3Kp110 $\alpha$ expression with clinicopathological characteristics and survival. The expression levels of PI3Kp110 $\alpha$ in OSCC were examined as a function of the clinical response to cetuximab therapy. Positive expression of PI3Kp110 $\alpha$ was significantly associated with stable disease (SD)/progressive disease (PD) in the clinical response $(\mathrm{P}<0.05)$. The overall response rate was $68.0 \%$, with 9 patients achieving $\mathrm{CR}$ and 8 achieving PR. The disease control rate was $84.0 \%$, which included 4 patients with SD (Table II). The cases of positive PI3Kp110 $\alpha$ expression originated from the following tissues: Tongue $(n=6)$, gingiva $(n=6)$, buccal mucosa $(n=1)$, and one primary intraosseous $(n=1)$, and no significant difference was found with respect to origin.
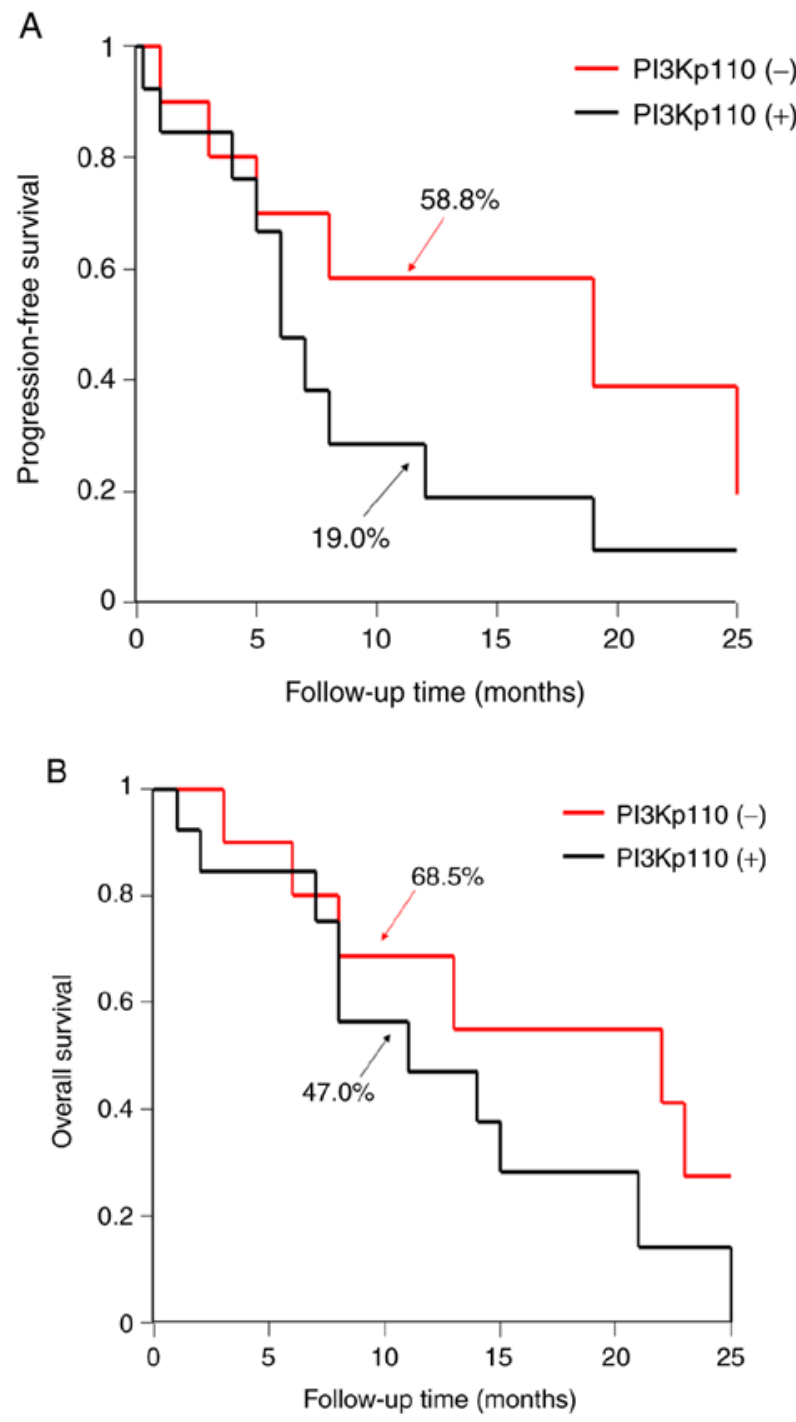

Figure 3. Association of the tumor best response following treatment with cetuximab and PI3Kp110 $\alpha$ expression. Kaplan-Meier curve of (A) progression-free survival rate and (B) overall survival rate. + , positive; -, negative. Percentages indicate the 1-year survival rates.

The 1-year progression-free survival (PFS) and overall survival (OS) rates, according to the expression level of PI3Kp110 $\alpha$, were determined. The Kaplan-Meier method revealed that the PI3Kp110 $\alpha$ high expression group had a lower PFS and OS compared with that in the low expression group. However, the 2-year PFS and OS between PI3Kp110 $\alpha$ high and low expression groups were equal (Fig. 3A and B).

Effect of PI3Kpl10 $\alpha$ expression on the cell migration and invasion potential of OSCC cell lines. At the protein level, the expression of PI3Kp110 $\alpha$ was evaluated using western blot analysis in three OSCC cell lines, following treatment with cetuximab (SAS, $813.7 \mathrm{nM}$; HSC-2, $82.4 \mathrm{nM}$; HSC-3, $952.2 \mathrm{nM}$ ). There was no significant difference between cetuximab sensitivity and PI3Kp110 $\alpha$ protein level; however, a high correlation was observed (Fig. 4A). For the migration and invasion indices, the correlation between protein expression level of PI3Kp110 $\alpha$ and migration and invasion potential were determined. There was a significant difference in the invasion 
A

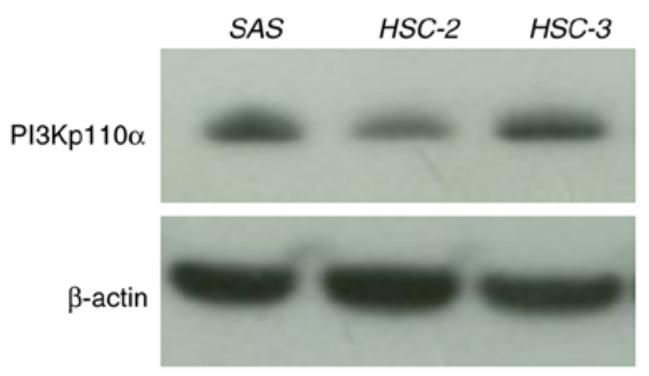

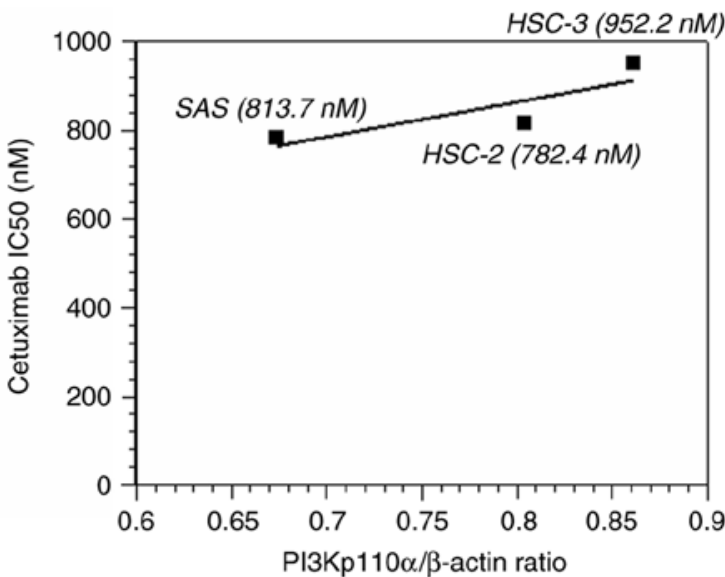

P/3Kp110 $\alpha / \beta$-actin ratio

\begin{tabular}{lcc}
\hline & & $\mathrm{IC} 50(\mathrm{nM})$ \\
\hline $\mathrm{PI} 3 \mathrm{Kp} 110 \alpha / \beta$-actin ratio & rs & 1.000 \\
& $P$ & 0.117 \\
\hline
\end{tabular}

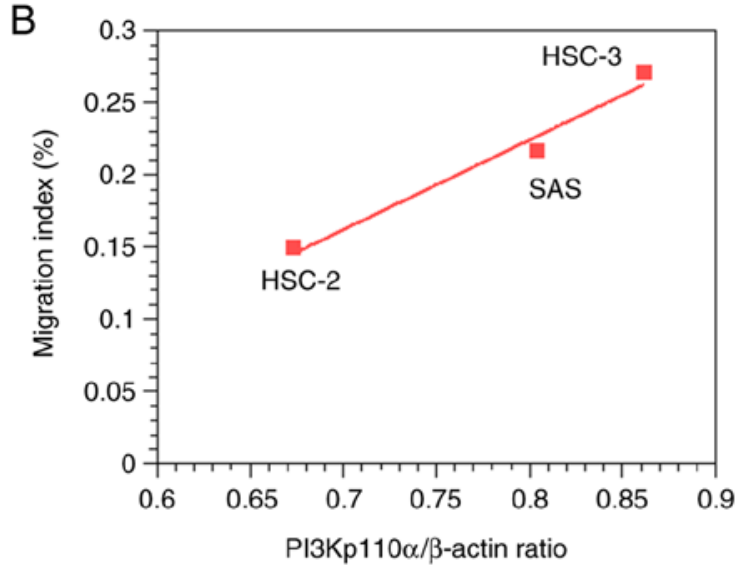

\begin{tabular}{ccc}
\hline & & Migration index \\
\hline PI3Kp110 $\alpha / \beta$-actin & rs & 0.987 \\
ratio & $P$ & 0.100 \\
\hline
\end{tabular}

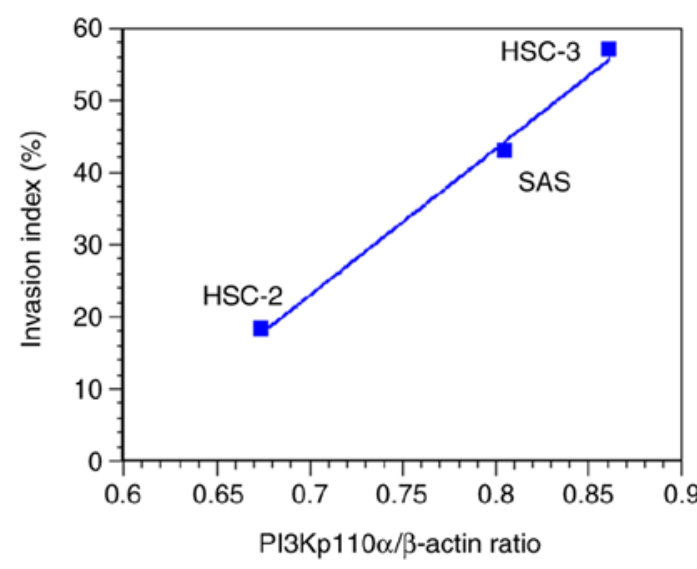

\begin{tabular}{ccc}
\hline & & Invasion index \\
\hline PI3Kp110 $\alpha / \beta$-actin & rs & 0.997 \\
ratio & $P$ & 0.042 \\
\hline
\end{tabular}

Figure 4. PI3Kp110 $\alpha$ protein expression. (A) Representative western blots of PI3Kp110 $\alpha$ expression in three OSCC cell lines (SAS, HSC-2, and HSC-3). The $\mathrm{PI} 3 \mathrm{Kp} 110 \alpha / \beta$-actin intensity is presented as mean $\pm \mathrm{SD}$ of triplicate experiments. (B) Association of PI3Kp110 $\alpha / \beta$-actin ratio with migration index (left) and invasion index (right). rs, correlation coefficient.

index ( $\mathrm{P}=0.042$; Fig. 4B), but not with migration index. As the HSC-3 cell line, which is a PIK3CA mutant cell line, exhibited high expression of PI3Kp110 $\alpha$ amongst the three cell lines, it was used for subsequent experiments.

The MTT assay revealed that alpelisib significantly inhibited cell proliferation in a dose-dependent manner in the HSC-3 cell line $(\mathrm{P}<0.05)$ (Fig. 5A). Moreover, western blot analysis revealed that alpelisib decreased the levels of PI3Kp110 $\alpha$, p-Akt, p-mTOR, p-p70S6K, and p-4EBP1 at the $\mathrm{IC}_{50}$ concentration $(23.54 \mu \mathrm{M})$, compared with that in the controls (Fig. 5B). In addition, the expression of E-cadherin was increased, while that of $\mathrm{N}$-cadherin was decreased.

Alpelisib and cetuximab alone or in combination decreases HSC-3 cell migration and invasion potential. Following treatment of HSC-3 cells with alpelisib or cetuximab alone or in combination there was a decrease in cell migration (Fig. 6A), which was found to be significantly different; the cell migration was significantly lower in the alpelisib or cetuximab only treatment groups and in combination compared with that in the control group (Fig. 6B; $\mathrm{P}<0.01$ ). In addition, there was a significant decrease in migration with the combined treatment group compared with that in cetuximab alone. No significant differences were found between the alpelisib or cetuximab only treatment groups.

Furthermore, there was also a decrease in cell invasion (Fig. 6C), which was also significant; cell invasion was significantly lower in the combination treatment group $(\mathrm{P}<0.01)$ and in the alpelisib or cetuximab only treatment groups $(\mathrm{P}<0.05)$ compared with that in the control group (Fig. 6D). 
A

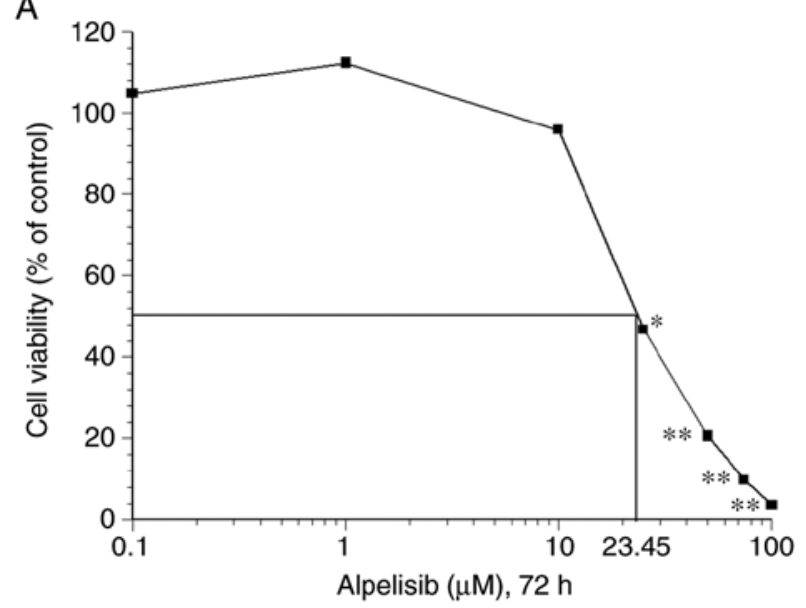

B
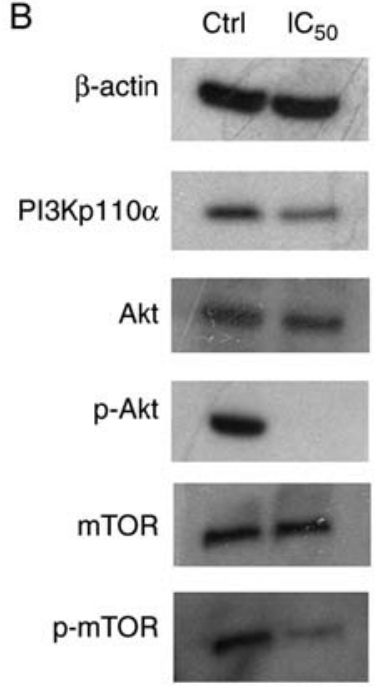
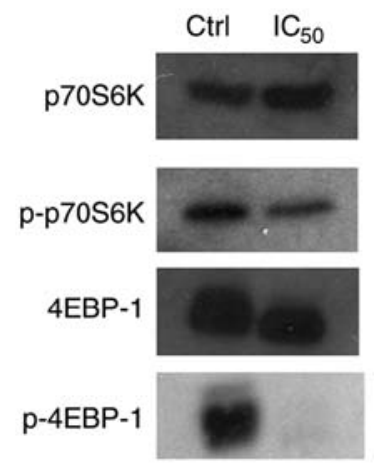

E-cadherin

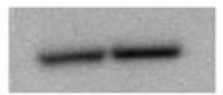

$\mathrm{N}$-cadherin

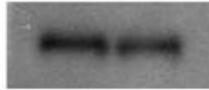

Figure 5. Effect of alpelisib on cell proliferation and PI3K signaling in HSC-3 OSCC cell line. (A) HSC-3 cells were exposed to various concentrations of alpelisib, (0.1 to $100 \mu \mathrm{M})$ and the percentage cell viability and drug concentration that inhibited cell growth by $50 \%\left(\mathrm{IC}_{50}\right)$ were calculated. (B) HSC-3 cell line was exposed to alpelisib at the $\mathrm{IC}_{50}$ concentrations and subsequently assessed for PI3K signaling and phosphorylated protein expression using western blot analysis. " $\mathrm{P}<0.05,{ }^{, *} \mathrm{P}<0.01$. Ctrl, control.

Effects of alpelisib and cetuximab alone or in combination in vivo. As alpelisib and cetuximab revealed antitumor effects in vitro, the antitumor potential was subsequently investigated in vivo effects. The weight of the mice in the control, cetuximab and alpelisib only treatment groups and in the combined treatment groups, at the end of the experiment, were $24.2,22.6,23.3$ and $22.8 \mathrm{~g}$, respectively, while the weight of the resected tumors were $1.8,0.14,0.34$ and $0.09 \mathrm{~g}$, respectively. The tumors in mice treated with cetuximab only grew markedly slower compared with that in the control group, and the tumors treated with alpelisib and cetuximab in combination also grew markedly slower compared with that in the control, alpelisib- and cetuximab-only treatment groups (Fig. 7A). However, there was no significant association between the type of treatment and tumor size. The protein expression levels of PI3Kp110 $\alpha$,EGFR, and p-mTOR was evaluated using immunohistochemistry in the excised tumors, and were highly expressed in the tumors from the control group. However, the expression levels of both EGFR and PI3Kp110 $\alpha$ were negative

in the majority of the tumor cells in the tumors treated with cetuximab-only. The expression of EGFR was strongly observed in the tumors treated with alpelisib only, whereas both PI3Kp110 $\alpha$ and p-mTOR were negative in the majority of the tumor cells. No expression of PI3Kp110 $\alpha$, EGFR, or p-mTOR expression was observed in the tumors treated with a combination of cetuximab and alpelisib (Fig. 7B).

\section{Discussion}

The NCCN Clinical Practice Guideline has suggested that systemic therapy (drug therapy), including cetuximab is the standard treatment for very advanced HNSCC; however, in some cases resistance to long-term administration of cetuximab develops, and new lesions can appear, including the brain $(3,6)$. The present study demonstrated that high protein expression of PI3Kp110 $\alpha$, which encodes the mutant PIK3CA gene, is associated with cetuximab resistance and survival. Furthermore, administration of alpelisib in combination with cetuximab, inhibits the Akt-mTOR pathway, providing additional antitumor effects, such as reduced growth in OSCC.

The clinicopathological results revealed that the protein expression of PI3Kp110 $\alpha$ was associated with response to cetuximab $(\mathrm{P}<0.05)$. In addition, patients with high expression of PI3Kp110 $\alpha$ had poor 1-year PFS and OS compared with that in patients with low expression. To the best of our knowledge, this is the first study to report the association of high PI3Kp110 $\alpha$ expression with cetuximab response. The point mutant PIK3CA gene has been associated with PFS, OS, and the clinical response to anti-EGFR antibodies, including cetuximab, in colorectal cancer (15). Moreover, somatic point mutations in exon 20 of PIK3CA are significantly associated with a low response to cetuximab in metastatic colorectal cancer (16). In HNSCC, treatment using cetuximab was not effective in seven patients with R/M HNSCC who also harbored the point mutant PIK3CA gene, as recurrence or metastasis was found (23). However, there was no association between patients with R/M OSCC, who also harbored the mutant PIK3CA gene and the cetuximab response. It is unclear whether it is appropriate to evaluate the same clinical response of cetuximab with the mutant PIK3CA gene and the protein expression of PI3Kp110 $\alpha$; although, overexpression of mutant PIK3CA gene notably increased the activation of the PI3K/Akt/mTOR pathway (13). Therefore, this suggests that high expression of PI3Kp110 $\alpha$ is a novel biomarker for the clinical response to cetuximab. With respect to prognosis, it was previously reported by our laboratory that cetuximab therapy provided additional benefits (such as improved 1-year OS) in patients with distant metastasis compared with patients receiving non-cetuximab therapy in 1-year, but not 2-year OS (5). It is difficult to maintain the therapeutic effect of cetuximab for $>2$ years; however, PI3Kp110 $\alpha$ could be a useful marker of one-year PFS and OS.

Biological evidence from the present study supports our hypothesis that the protein expression of PI3Kp110 $\alpha$ extracted from OSCC cell lines is associated with the cetuximab response and is significantly associated with invasive potential. Alpelisib was selected to inhibit the PI3Kp110 $\alpha$ protein, as it is a single agent with proven effectiveness by inhibiting proliferation, apoptosis and inducing cell cycle arrest in HNSCC in a preclinical study (24). In the preclinical study of HNSCC, 

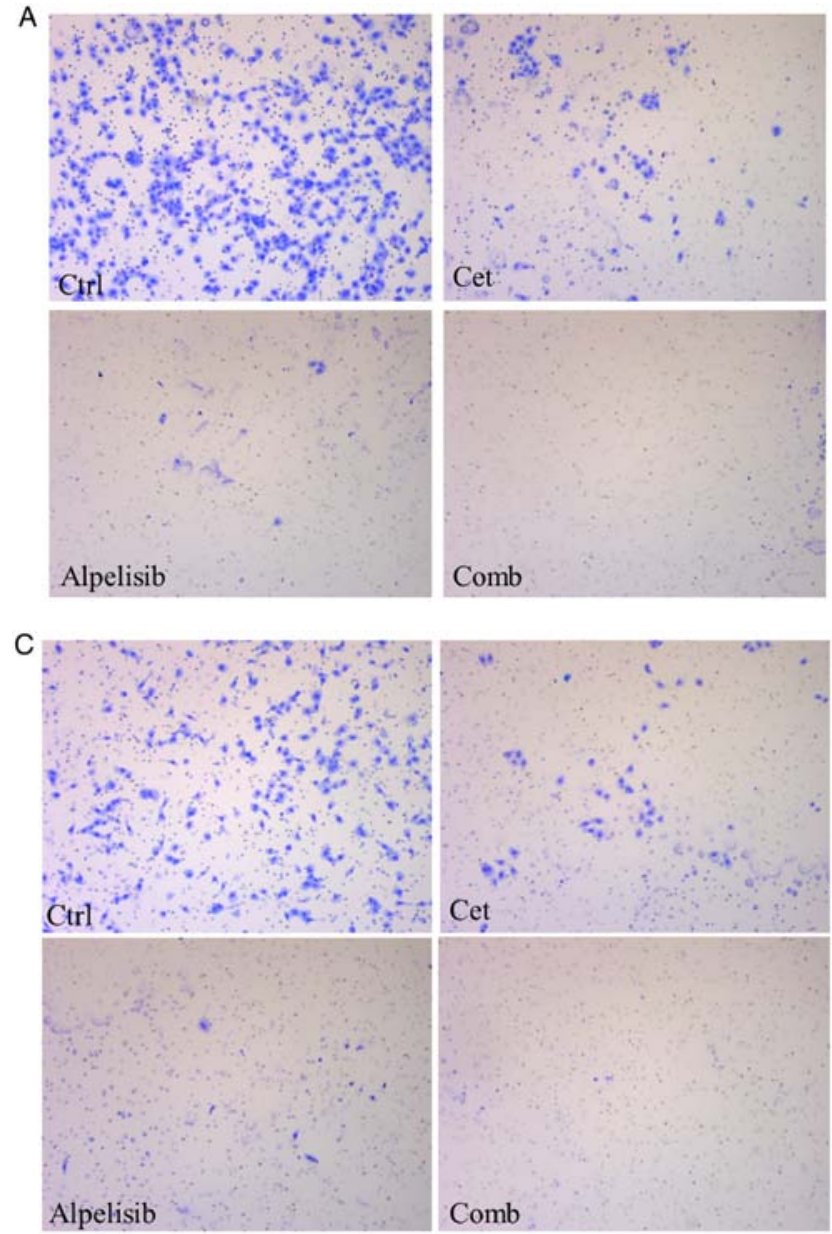
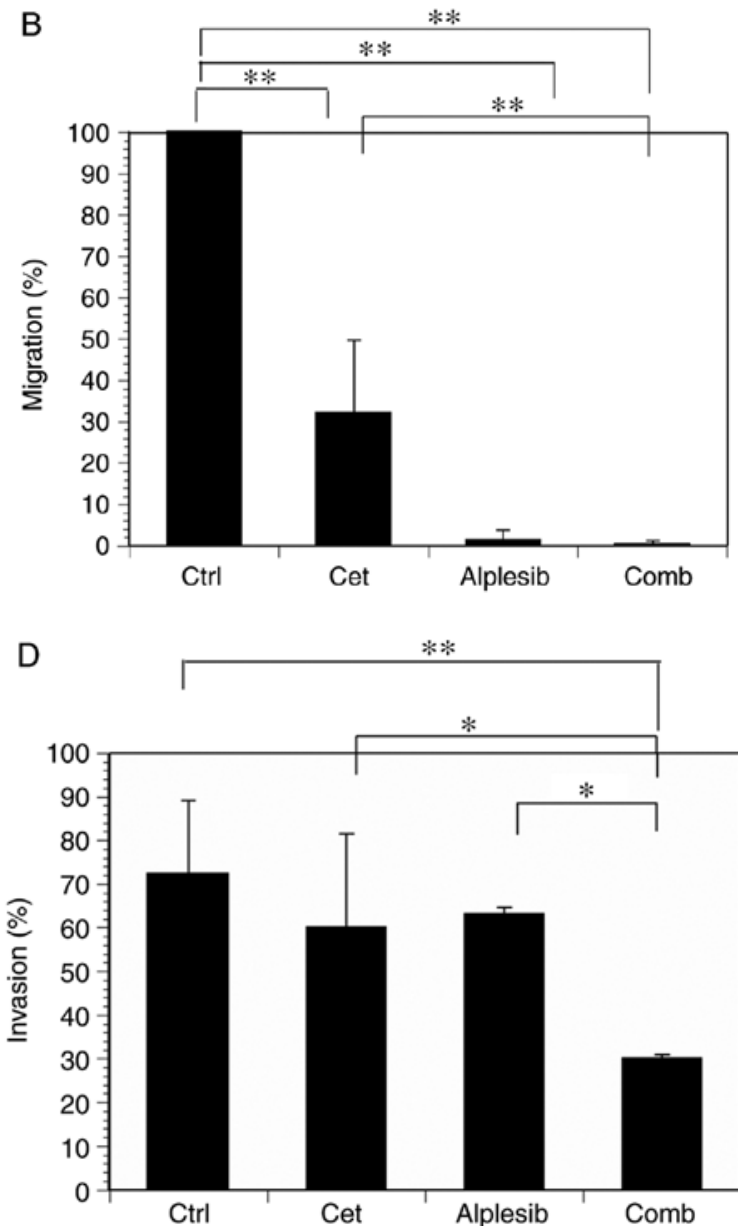

Figure 6. Effect of treatment with alpelisib or cetuximab only and in combination on migration and invasion of HSC-3 OSCC cell line. (A) Representative cell migration images of HSC-3 cells and (B) the percentage of migrating cells. Significant decreases in the migration index following treatment with alpelisib and cetuximab alone and in combination were found compared with that in the control group. (C) Representative cell invasion images of HSC-3 cells and (D) the percentages of invading cells. Significant decreases in cell invasion following combination treatment compared with that in the control and single treatment groups. ${ }^{~} \mathrm{P}<0.05,{ }^{* *} \mathrm{P}<0.01$. Ctrl, control; cet, cetuximab; comb, combination.
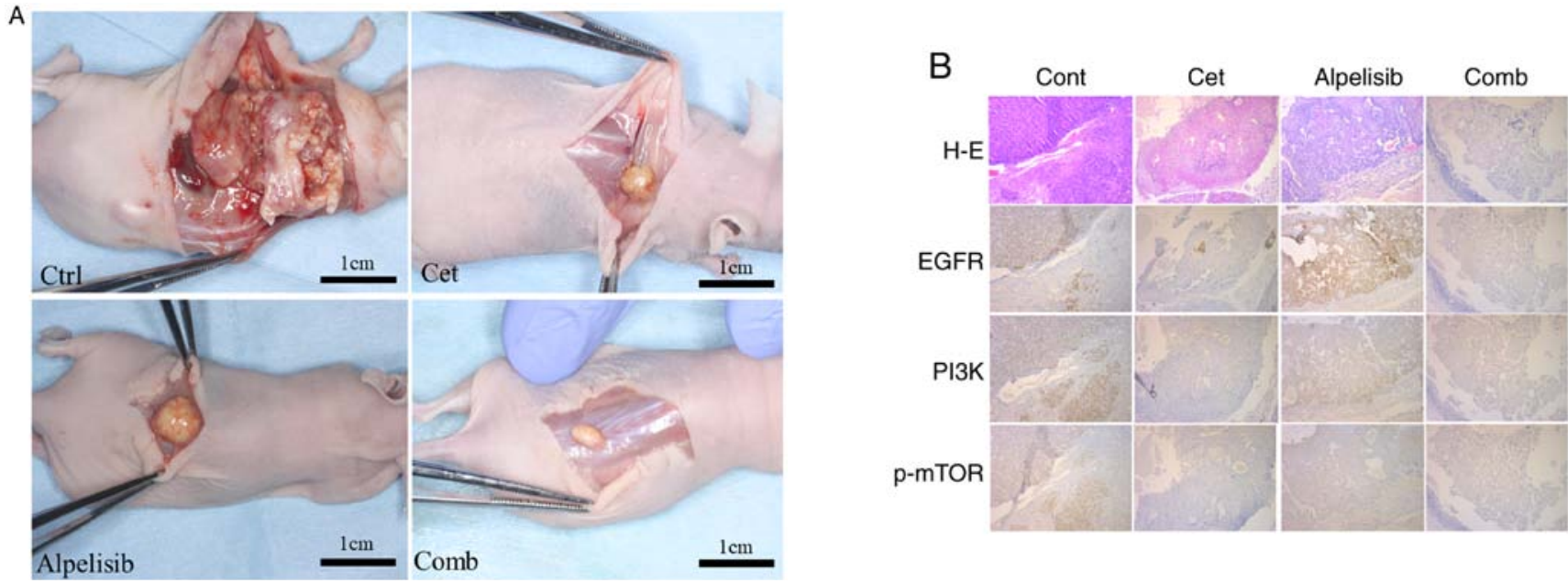

Figure 7. Effects of alpelisib and cetuximab alone or in combination in vivo. (A) Representative images of the HSC-3 xenograft tumors in mice treated with either control (saline-treated), cetuximab-only, alpelisib-only, or in combination. (B) Representative images of paraffin-embedded sections of HSC-3 tumors treated with either control, cetuximab-only, alpelisib-only, and in combination in vivo stained with H\&E or EGFR) PI3Kp110 $\alpha$, and mTOR Ser2448 Magnification, x100. H\&E, hematoxylin and eosin; cont, control; cet, cetuximab; comb, combination.

alpelisib was shown to overcome cetuximab resistance in HNSCC (25). In addition, alpelisib treatment reduced Akt activation and suppressed tumor growth in HNSCC, in vitro and in vivo $(25,26)$. In the present study the HSC-3 OSCC cell 
line were found to be sensitive to the anti-proliferative effects of alpelisib. Moreover, there was also a decrease in phosphorylation of downstream markers of the PI3K-Akt pathway in a dose-dependent manner. In particular, alpelisib-mediated suppression of the epithelial-to-mesenchymal transition (EMT), which was confirmed using western blot analysis. Kimura et al (27) reported that cetuximab-resistant cell lines (including HOC313) increased the mRNA and protein expression levels of EMT-associated genes (such as E-cadherin, $\mathrm{N}$-cadherin, vitmentin and Snail). Schmitz et al (28) also determined the association between the increase in protein expression of EMT markers (ZEB1, TWIST1, TWIST2, LEF1, VIM, SNAI1 and SNAI2) and cetuximab resistance using 20 pre- and post-cetuximab treatment HNSCC biopsy specimens $(\mathrm{P}<0.05)$. Moreover, in our previous study there was a case of distant metastasis and the appearance of new lesions following long-term cetuximab therapy was observed (6). In addition, based on the immunohistochemical staining of recurrent tumors in this case before and after long-term cetuximab administration, cetuximab resistance may be associated with EMT (6). These reports suggest that cetuximab-resistant cells possess high invasive and migration potential (associated with EMT) and that inhibition of PI3Kp $110 \alpha$ might inhibit the potential of cetuximab-resistant cells to undergo EMT in OSCC.

We hypothesized that cetuximab and alpelisib would have additive anti-cancer effects in vitro, through selective blocking of the extracellular ligand-binding domain of EGFR by cetuximab, while alpelisib competed for the intracellular catalytic site of PI3K. It has been reported that alpelisib in combination with cetuximab had an overall response rate of $11 \%$ and a disease control rate of $54 \%$ in cetuximab-resistant HNSCC (25); however, OSCC was not included in this study. The EXTREME trial revealed that the benefit of including cetuximab to platinum-based chemotherapy for patients with HNSCC was more effective in patients with OSCC. However, the results should be interpreted with caution, as this does not suggest that OSCCs would be the only HNSCC tumor types to respond to this type of therapy (2). Therefore, studies with OSCC alone are required. In the present study, both cetuximab and alpelisib inhibited cell invasion and migration potential, and their combined treatment resulted in additive inhibition of OSCC migration and invasion potential compared with that in either agent alone. In our previous study the inhibition of the PI3K downstream pathway decreased the migration and invasion ability in the HSC-3 cell line (29). Therefore, combined treatment with alpelisib and cetuximab was effective in OSCC invasion in vitro.

The combined treatment of alpelisib and cetuximab markedly inhibited OSCC progression in vivo compared with that in either agent alone; however, there was no significant association with tumor size. This could be due to the antitumor effects of alpelisib or cetuximab alone in the xenograft tumor, which was evident from the decreased protein expression levels of PI3Kp110 $\alpha$ from immunohistochemistry analysis.

The immunohistochemical study is retrospective nature and includes a small number of cases from a single institution, which is a limitation to the present study. Therefore, an intergroup study with an increased number of cases is required.

In conclusion, the results of the present study suggest that the PI3K pathway plays an important role in cetuximab-resistant
OSCC, and that combined treatment with alpelisib and cetuximab may overcome cetuximab resistance. Previous studies have revealed that, nivolmab, an anti-programmed cell death protein 1 (PD-1) monoclonal antibody, has been recommended as another treatment option for R/M HNSCC $(3,30)$. However, the overall response rate is not high, thus additional treatment options are required. Therefore, novel systemic drug treatment regimens that include $\mathrm{PI} 3 \mathrm{~K}$ inhibitors are expected to further improve the survival of patients with cetuximab-resistant OSCC in the future.

\section{Acknowledgements}

Not applicable.

\section{Funding}

This study was partially supported by the Ministry of Education, Culture, Sports, Science and Technology, Japan (grant no 17K17267).

\section{Availability of data and materials}

The datasets used and/or analyzed during the current study are available from the corresponding author on reasonable request.

\section{Authors' contributions}

TN, SY and MU designed the study. HT, TN, KOk, KF and KOm performed the experiments. HT, TN and KOk analyzed the data. HT, TN and MU wrote the manuscript. All authors read and approved the final manuscript.

\section{Ethical approval and consent to participate}

This study was approved by the independent ethics committee of Nagasaki University Hospital (approval no. 19081915).

\section{Patient consent for publication}

Not applicable.

\section{Competing interests}

The authors declare that they have no competing interests.

\section{References}

1. Ho AS, Kraus DH, Ganly I, Lee NY, Shah JP and Morris LG: Decision making in the management of recurrent head and neck cancer. Head Neck 36: 144-151, 2014.

2. Vermorken JB, Mesia R, Rivera F, Remenar E, Kawecki A, Rottey S, Erfan J, Zabolotnyy D, Kienzer HR, Cupissol D, et al: Platinum-based chemotherapy plus cetuximab in head and neck cancer. N Engl J Med 359: 1116-1127, 2008.

3. National Comprehensive Cancer Network (NCCN): NCCN

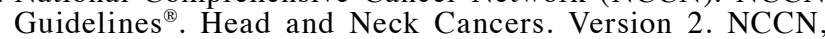
Plymouth Meeting, PA, 2019

4. Yanamoto S, Umeda M, Kioi M, Kirita T, Yamashita T, Hiratsuka H, Yokoo S, Tanzawa H, Uzawa N, Shibahara T, et al: Multicenter retrospective study of cetuximab plus platinum-based chemotherapy for recurrent or metastatic oral squamous cell carcinoma. Cancer Chemother Pharmacol 81: 549-554, 2018. 
5. Naruse T, Yanamoto S, Matsushita Y, Sakamoto Y, Morishita K, Ohba S, Shiraishi T, Yamada SI, Asahina I and Umeda M: Cetuximab for the treatment of locally advanced and recurrent/metastatic oral cancer: An investigation of distant metastasis. Mol Clin Oncol 5: 246-252, 2016.

6. Naruse T, Yanamoto S, Matsushita Y, Sakamoto Y, Morishita K, Ohba S, Shiraishi T, Yamada SI, Asahina I and Umeda M: Lower gingival squamous cell carcinoma with brain metastasis during long-term cetuximab treatment: A case report. Oncol Lett 15: 7158-7162, 2018.

7. Bonner JA, Harari PM, Giralt J, Azarnia N, Shin DM, Cohen RB, Jones CU, Sur R, Raben D, Jassem J, et al: Radiotherapy plus cetuximab for squamous-cell carcinoma of the head and neck. N Engl J Med 354: 567-578, 2006

8. Hitt R, Irigoyen A, Cortes-Funes H, Grau JJ, García-Sáenz JA and Cruz-Hernandez JJ; Spanish Head and Neck Cancer Cooperative Group (TTCC): Phase II study of the combination of cetuximab and weekly paclitaxel in the first-line treatment of patients with recurrent and/or metastatic squamous cell carcinoma of head and neck. Ann Oncol 23: 1016-1022, 2012.

9. Wang Z, Martin D, Molinolo AA, Patel V, Iglesias-Bartolome R, Degese MS, Vitale-Cross L, Chen Q and Gutkind JS: mTOR co-targeting in cetuximab resistance in head and neck cancers harboring PIK3CA and RAS mutations. J Natl Cancer Inst 106 dju215, 2014.

10. Swick AD, Prabakaran PJ, Miller MC, Javaid AM, Fisher MM, Sampene E, Ong IM, Hu R, Iida M, Nickel KP, et al: Co-targeting mTORC and EGFR signaling as a therapeutic strategy in HNSCC. Mol Cancer Ther 16: 1257-1268, 2017.

11. Rampias T, Giagini A, Siolos S, Matsuzaki H, Sasaki C, Scorilas A and Psyrri A: RAS/PI3K crosstalk and cetuximab resistance in head and neck squamous cell carcinoma. Clin Cancer Res 20: 2933-2946, 2014.

12. Kozaki K, Imoto I, Pimkhaokham A, Hasegawa S, Tsuda H, Omura $\mathrm{K}$ and Inazawa J: PIK3CA mutation is an oncogenic aberration at advanced stages of oral squamous cell carcinoma. Cancer Sci 97: 1351-1358, 2006.

13. Ligresti G, Militello L, Steelman LS, Cavallaro A, Basile F, Nicoletti F, Stivala F, McCubrey JA and Libra M: PIK3CA mutations in human solid tumors: Role in sensitivity to various therapeutic approaches. Cell Cycle 8: 1352-1358, 2009.

14. Chang YS, Hsu HT, Ko YC, Yeh KT, Chang SJ, Lin CY and Chang JG: Combined mutational analysis of RAS, BRAF, PIK3CA, and TP53 genes in Taiwanese patients with oral squamous cell carcinoma. Oral Surg Oral Med Oral Pathol Oral Radiol 118: 110-116.e1, 2014.

15. Sartore-Bianchi A, Martini M, Molinari F, Veronese S, Nichelatti M, Artale S, Di Nicolantonio F, Saletti P, De Dosso S and Mazzucchelli L: PIK3CA mutations in colorectal cancer are associated with clinical resistance to EGFR-targeted monoclonal antibodies. Cancer Res 69: 1851-1857, 2009.

16. De Roock W, Claes B, Bernasconi D, De Schutter J, Biesmans B, Fountzilas G, Kalogeras KT, Kotoula V, Papamichael D Laurent-Puig P, et al: Effects of KRAS, BRAF, NRAS, and PIK3CA mutations on the efficacy of cetuximab plus chemotherapy in chemotherapy-refractory metastatic colorectal cancer: A retrospective consortium analysis. Lancet Oncol 11: 753-762, 2010.

17. Sok MZ, Zavrl M, Greif B and Srpčič M: Objective assessment of WHO/ECOG performance status. Support Care Cancer 27: 3793-3798, 2019.
18. Nishino M, Jackman DM, Hatabu H, Yeap BY, Cioffredi LA, Yap JT, Jänne PA, Johnson BE and Van den Abbeele AD: New response evaluation criteria in solid tumors: Revised RECIST guideline version 1.1. Eur J Cancer (Suppl 6): S13, 2008.

19. Pinborg JJ, Reichart PA, Smith CJ and van der Waal I: Histological typing of cancer and precancer of the oral mucosa. 2nd ed. Berlin, Germany: Springer; 1997.

20. Wright JM and Vered M: Update from the 4th edition of the world health organization classification of head and neck tumours: Odontogenic and maxillofacial bone tumors. Head Neck Pathol 11: 68-77, 2017.

21. Bryne M, Boysen M, Alfsen CG, Abeler VM, Sudb $\varnothing$ J, Nesland JM, Kristensen GB, Piffko J and Bankfalvi A: The invasive front of carcinomas. The most important area for tumour prognosis? Anticancer Res 18: 4757-4764, 1998.

22. Vis DJ, Bombardelli L, Lightfoot H, Iorio F, Garnett MJ and Wessels LF: Multilevel models improve precision and speed of IC50 estimates. Pharmacogenomics 17: 691-700, 2016.

23. Jimeno A, Shirai K, Choi M, Laskin J, Kochenderfer M, Spira A, Cline-Burkhardt V, Winquist E, Hausman D, Walker L and Cohen RB: A randomized, phase II trial of cetuximab with or without PX-866, an irreversible oral phosphatidylinositol 3-kinase inhibitor, in patients with relapsed or metastatic head and neck squamous cell cancer. Ann Oncol 26: 556-561, 2015.

24. Keam B, Kim S, Ahn YO, Kim TM, Lee SH, Kim DW and Heo DS: In vitro anticancer activity of PI3K alpha selective inhibitor BYL719 in head and neck cancer. Anticancer Res 35: 175-182, 2015.

25. Munster P, Elkabets M, Gilbert J, Razak ARA, Ahn MJ, Yen CJ, Lee SH, Wang HM, Herpen C and Lim WT: Abstract A46: Inhibition of PIK3CA with BYL719 can overcome resistance to cetuximab in squamous cell carcinoma of the head and neck (SCCHN). Mol Cancer Ther 14 (Suppl 7): A46-A46, 2015.

26. Meister KS, Godse NR, Khan NI, Hedberg ML, Kemp C, Kulkarni S, Alvarado D, LaVallee T, Kim S, Grandis JR and Duvvuri U: HER3 targeting potentiates growth suppressive effects of the PI3K inhibitor BYL719 in pre-clinical models of head and neck squamous cell carcinoma. Sci Rep 9: 9130, 2019.

27. Kimura I, Kitahara H, Ooi K, Kato K, Noguchi N, Yoshizawa K, Nakamura $\mathrm{H}$ and Kawashiri S: Loss of epidermal growth factor receptor expression in oral squamous cell carcinoma is associated with invasiveness and epithelial-mesenchymal transition. Oncol Lett 11: 201-207, 2016.

28. Schmitz S, Bindea G, Albu RI, Mlecnik B and Machiels JP: Cetuximab promotes epithelial to mesenchymal transition and cancer associated fibroblasts in patients with head and neck cancer. Oncotarget 6: 34288-34299, 2015.

29. Naruse T, Yanamoto S, Okuyama K, Yamashita K, Omori K, Nakao Y, Yamada SI and Umeda M: Therapeutic implication of mTORC2 in oral squamous cell carcinoma. Oral Oncol 65: 23-32, 2017.

30. Ferris RL, Blumenschein G Jr, Fayette J, Guigay J, Colevas AD, Licitra L, Harrington K, Kasper S, Vokes EE, Even C, et al: Nivolumab for recurrent squamous-cell carcinoma of the head and neck. N Engl J Med 375: 1856-1867, 2016.

This work is licensed under a Creative Commons Attribution-NonCommercial-NoDerivatives 4.0 International (CC BY-NC-ND 4.0) License. 